\title{
Chitosan/hyaluronic acid multilayer films are biocompatible substrate for Wharton's jelly derived stem cells
}

\author{
Hana Dennaoui ${ }^{1}$, Eliane Chouery ${ }^{2}$, Hassan Rammal $^{3}$, Ziad Abdel-Razzak ${ }^{1}$, Chaza Harmouch ${ }^{1}$ \\ ${ }^{1}$ Laboratory of Applied Biotechnology: Biomolecules, Biotherapies and Bioprocesses, AZM Centre for Biotechnology research and its Applications, \\ Doctoral School of Science and Technology, Lebanese University, Tripoli, Lebanon; ${ }^{2}$ Medical Genetics Unit, Faculty of medicine, Saint Joseph \\ University (USJ), Beirut, Lebanon; ${ }^{3}$ Equipe de Recherche sur les relations Matrice extracellulaire-Cellules (ERRMECe), Institut des Materiaux, \\ Maison International de la Recherche, Universite de Cergy-Pontoise, 95000 Neuville sur Oise, France \\ Contributions: (I) Conception and design: H Dennaoui, C Harmouch; (II) Administrative support: Z Abdel-Razzak, E Chouery; (III) Provision of \\ study materials or patients: H Dennaoui; (IV) Collection and assembly of data: H Dennaoui; (V) Data analysis and interpretation: H Dennaoui, C \\ Harmouch, Z Abdel-Razzak; (VI) Manuscript writing: All authors; (VII) Final approval of manuscript: All authors. \\ Correspondence to: Chaza Harmouch. Laboratory of Applied Biotechnology: Biomolecules, Biotherapies and Bioprocesses, AZM Centre for \\ Biotechnology research and its Applications, Doctoral School of Science and Technology, Lebanese University, El Mitein Street, Tripoli, Lebanon. \\ Email: shako_87@hotmail.com.
}

Background: Discovery of mesenchymal stem cells (MSCs) in various adult human tissues opened the way to new therapeutic strategies involving tissue engineering from these cells. More recently, vascular substitutes have opened the era of vascular engineering by making replacement vessels from purely biological material. The objective of our study was to create a vascular substitute from MSCs using a multilayer polyelectrolyte film based on natural polymers (Chitosan and Hyaluronic Acid).

Methods: Biocompatibility and cellular behavior were evaluated in this study using MSCs from the Wharton's jelly (WJ) of human umbilical cords. WJ-MSCs adherence was assessed and cells morphology was investigated by Scanning Electron Microscopy (SEM) and actin visualization (Phalloidin).

Results: The number of WJ-MSCs seeded on the (CHI/HA $)_{10}$ films was greater than the number of cells seeded on the collagen, as the spectrophotometric measurement showed a large cell proliferation on (CHI/ $\mathrm{HA})_{10}$ in comparison with collagen. After adhesion, WJ-MSCs showed a fibroblastic morphology on CHI/ HA as for control (collagen I). These results were confirmed by cytoskeleton staining.

Conclusions: The biocompatibility of WJ-MSCs and (CHI/HA $)_{10}$ showed the possibility to combine the use of WJ-MSCs and (CHI/HA $)_{10}$ films in vascular tissue engineering.

Keywords: Mesenchymal stem cells (MSCs); tissue engineering; multilayer films

Received: 09 June 2018; Accepted: 28 November 2018; Published: 20 December 2018.

doi: $10.21037 /$ sci.2018.12.02

View this article at: http://dx.doi.org/10.21037/sci.2018.12.02

\section{Introduction}

Bone marrow, the first source of mesenchymal stem cells (MSCs) described in adult humans, is the most commonly used in tissue engineering. However, availability of human marrow, invasiveness of its collection and the immunogenicity of cells after differentiation make its use very controversial. Since 2004, focus was on a new source of MSCs, the Wharton's jelly (WJ) of the human umbilical cord. Several studies showed better proliferation of WJderived MSCs in comparison with bone marrow-derived MSCs. Diverse extracellular matrix types, including fibronectin and type I collagen, were used in order to improve MSCs adhesion and differentiation in vitro. Development of other matrix types, such as the multilayer polyelectrolyte films, is likely to improve proliferation and differentiation of MSCs. Moreover, less clinical risks are expected with multilayers based on biocompatible 
natural polyanion polymers (e.g., hyaluronic acid, HA) and polycation polymers (e.g., chitosan, CHI). Layer by layer deposition of polyelectrolytes is a versatile technique which is beginning to be explored as smart coating for tissue engineering application (1). In the vascular regenerative field, this approach is often employed to promote vascular cell adhesion $(2,3)$, to differentiate stem cells into vascular cells $(4,5)$ to inhibit thrombus formation and in vitro smooth muscle cell dedifferentiation $(2,6)$, and to embed bioactive molecules in the film architecture to design drug delivery material (7). The most studied film from synthetic polyelectrolytes, such as poly-(styrene sulfonate) and poly(allylamine hydrochloride), are relatively stiff and thus favour cell adhesion and proliferation $(8,9)$. However, they are not approved by the US and European health authorities due their potential inflammatory risks in clinical use (6). Designing polyelectrolyte multilayer (PEM) films using natural polyelectrolytes (polysaccharides or proteins) is then of great interest for medical applications. PEMs made from Chitosan and Hyaluronic Acid are highly hydrated and often behave as gel-like with a weak stiffness (1,9-12). They have been described as poorly adhesive for chondrosarcoma and osteoblastic cell line $(13,14)$ but enabled good human fibroblasts and stem cells adhesion and proliferation (15) through specific HA-receptor (i.e., CD44) (11).

According to the International Society for Cellular Therapy, the minimal features to define MSCs are their growth pattern in vitro (plastic-adherent cells), the specific surface antigen expression (CD73, CD90 and CD105, in the absence of lineage commitment markers such as CD14, CD19, CD34, CD45 and HLA-DR) and multilineage potential (ability to differentiate into osteoblasts, adipocytes and chondroblasts in vitro) (16). These unique characteristics of MSCs are advantageous in terms of therapeutic applications (17-19). The main source for MSCs is the bone marrow but recently perinatal tissues, like the umbilical WJ, have been recognized as an excellent source to isolate MSCs. WJ can be easily obtained and WJMSCs are more primitive MSCs than those isolated from adult tissues and do not express the major histocompatibility complex (MHC) class II (HLA-DR) antigens (20). Different studies showed that WJ-MSCs were still viable and were not rejected four months after transplantation as xenografts without immune suppression treatment, suggesting that they are a potential cell source for transplantation $(16,21)$.

The objective of this work was to evaluate the biocompatibility of CHI/HA PEMs for WJ-MSCs. We showed that CHI/HA films promote WJ-MSCs adhesion and proliferation. WJ-MSCs expressed MSCs markers and showed fibroblastic-like morphology. Our findings suggest that $\mathrm{CHI} / \mathrm{HA}$ films could be used in tissue engineering approaches.

\section{Methods}

\section{Polyelectrolytes multilayer films}

Hyaluronic acid solution $(0.2 \mathrm{mg} / \mathrm{mL}$ in $\mathrm{NaCl} 0.15 \mathrm{M})$ and chitosan solution $(0.2 \mathrm{mg} / \mathrm{mL}$ in $\mathrm{NaCl} 0.15 \mathrm{M} / \mathrm{HCl} 2 \mathrm{mM})$ were used to build the polyelectrolyte multilayers. Reagents were obtained from commercial sources and used without any further purification. Chitosan low molecular weight and hyaluronan (HA, MW $=200 \mathrm{kDa}$ ) were obtained from Sigma Aldrich (Germany).

Each experiment was preceded by a cleaning step of the cover glasses as follow: 15 min with sodium dodecyl sulfate $1 \%$ (Sigma Aldrich, Germany) at $100{ }^{\circ} \mathrm{C}$, extensive ultrapure water rinse, $15 \mathrm{~min}$ at $100{ }^{\circ} \mathrm{C}$ with $10^{-1} \mathrm{M} \mathrm{HCl}$ and finally cover glasses were thoroughly rinsed with ultrapure water. Coverslips were incubated in CHI solution for $5 \mathrm{~min}$, thoroughly washed in $\mathrm{NaCl}(0.5 \mathrm{M})$ and then incubated in HA solution for $5 \mathrm{~min}(\mathrm{CHI}-\mathrm{HA})_{10}$ films were built up after 20 alternate depositions of polycation and polyanion layers. The type I collagen $(100 \mu \mathrm{g} / \mathrm{mL}$ from BD) was used as positive control for cellular adhesion. The collagen solution was added on the coverslips and incubated for 1 hour at room temperature. Then, the solution was carefully aspirated and glasses surface was rinsed 3 times with serum-free $\alpha$-MEM.

\section{Stem cell isolation and culture}

This study was approved by the ethical committee of Al Hanan Hospital-Tripoli, Lebanon. Twenty five fresh human umbilical cords were obtained after full-term birth. Umbilical cord vessels were removed manually from cord segments, and the exposed WJ was cut into very small pieces or explants which were cultured in $\alpha$-MEM (Sigma Aldrich,) supplemented with $10 \%$ decomplemented fetal bovine serum (FBS), $2 \mathrm{mM}$ L-glutamine, $100 \mathrm{IU} / \mathrm{mL}$ Penicillin/streptomycin and $2.5 \mathrm{mg} / \mathrm{mL}$ Fungizon ${ }^{\circledR}$ (Sigma Aldrich, ) at $37{ }^{\circ} \mathrm{C}$ and in $5 \% \mathrm{CO}_{2}$. After migration of $\mathrm{WJ}$ MSCs from the explants and their adhesion to the culture dish matrix, explants were removed and cell culture was maintained with a medium change every other day until their characterization after the $3^{\text {rd }}$ passage $(\mathrm{P} 3)$. 


\section{Characterization of W7-MSCs by flow cytometry}

WJ-MSCs were characterized at $3^{\text {rd }}$ passage by flow cytometry (FACSCalibur; BD Bioscience) through the expression of MSC's markers cluster of differentiation, CD73 (Ecto-5'-nucleotidase), CD90 (Thymocyte differentiation antigen-1) and CD105 (Endoglin), hematopoietic markers CD34 and CD45 (Leukocyte common antigen, Ly-5); using mouse anti-human CD45(HI30) fluorescein isothiocyanate FITC-conjugated antibody (BD Bioscience), phycoerythrin PE-conjugated mouse anti-human CD34-(581), CD73-(AD2), CD90(5E10) and CD105-(5N6) antibodies (BD Pharmingen, France) and appropriate isotype controls (mouse IgG1-PE, IgG1-FITC and IgG1-HLA-DR (BD Pharmingen, France). Briefly, adherent WJ- and BM-MSCs were detached using trypsin/ethylenediaminetetraacetic acid (EDTA) and incubated for 1 hour at room temperature with appropriate conjugated antibodies. At least 10,000 events were acquired on BD FACS Calibur ${ }^{\circledR}$ flow cytometer, and the results were analyzed using WinMDI v2.8 software.

\section{Evaluation of W7-MSCs adberence on CHI/HA films}

WJ-MSCs adherence on (CHI/HA $)_{10}$ films and type I collagen substrates was determined by Crystal violet 0.1 M (Sigma Aldrich, Germany), a dye that interacts with intracellular proteins. At $4^{\text {th }}$ passage and after non-adherent cells were discarded by PBS washes and the adherent cells were stained with $150 \mu \mathrm{L}$ of crystal violet for $15 \mathrm{~min}$ at $37^{\circ} \mathrm{C}$. After extraction of the dye in SDS/PBS (1\%), extent of cell adhesion was quantified by measuring the optical density of the eluted crystal violet at $570 \mathrm{~nm}$ by a fluorescence spectrophotometer (Varioskan Flash, Thermo scientific). The eluted crystal violet of pre-permeabilized cells with Triton (X100)/PBS (0.1\%) was used as a negative control (22).

WJ-MSCs seeded on (CHI/HA $)_{10}$ films were incubated with DAPI (0.4\%) (Sigma Aldrich, Germany) for $5 \mathrm{~min}$ at room temperature. Different fields were observed by fluorescence microscopy (493 nm, Zeiss microscopy, Japan) and then visual nuclei counting was compared in photographs.

\section{Evaluation of CHI/HA films integrity}

F-actin cytoskeleton was labelled using Phalloidin ${ }^{\circledR}$ coupled with Alexa ${ }^{\circledR} 488$ (Sigma Aldrich, Germany). After 2 weeks of culture, WJ-MSCs were fixed with $4 \%$ paraformaldehyde for $15 \mathrm{~min}$, permeabilized with PBS/Triton X-100 (0.1\%) for 15 min and blocked with PBS/BSA (1\%). Cells were then incubated for $45 \mathrm{~min}$ at $37^{\circ} \mathrm{C}$ with Phalloidin ${ }^{\circledR}(1 / 100)$. After two washes with PBS, nuclei were counterstained using DAPI for $5 \mathrm{~min}$ at room temperature. The labeled cells were mounted on slides to be observed by fluorescence microscopy (Zeiss microscopy, $\times 20$ magnification) using the $\left(493_{\mathrm{Ex}} / 538_{\mathrm{Em}}\right)$ spectral line.

WJ-MSCs were fixed in $2.5 \%$ glutaraldehyde (Sigma Aldrich, Germany) followed by two washing steps with PBS. Then, they are dehydrated by successive passages in alcohol solutions of increasing concentration: $(50 \%, 70 \%, 95 \%$ and $100 \%$; 10 minutes for each concentration). Cells are dried with HMDS (Hexamethyldisilazane, Sigma Aldrich, Germany) and samples are plated with gold palladium in a cathodic evaporator JEOL JFC-1100 ION SPUTTER $(8 \mathrm{~mA}$ and $1.2 \mathrm{kV}$ ) for observation. After drying, the specimens were directly put on a carbon pad of a SEMholder. Scanning Electron Microscopy (SEM) was performed with a JEOL Phillips microscope (JEOL 5400 LV).

\section{Statistical analysis}

Data are presented as mean \pm SEM for each condition. Each experiment was repeated independently three times $(n=3)$. Pairwise comparison was performed using one-factor ANOVA with Fisher correction (Statview IVs, Abacus Concepts Inc., Berkley, CA). Differences were considered significant if $\mathrm{P}<0.05$.

\section{Results}

\section{Characterization of W7-MSCs by flow cytometry}

Results are representative of those obtained using cells from umbilical cords of three distinct donors. Flow cytometry data showed that $99 \%$ of $\mathrm{WJ}-\mathrm{MSCs}$ were positive for both CD73 and CD90 expression; and 98\% of these cells were positive for CD105 expression. WJ-MSCs displayed a homogeneous fibroblast-like phenotype with no expression of CD34 (5\%), CD45 (1\%) (hematopoietic lineage markers) and HLA-DR (MHC-class II) (1\%) (Figure 1). Because WJ-MSCs expressed MSCs markers and did not express hematopoietic markers, they may be all considered of mesenchymal origin.

\section{Evaluation of W7-MSCs adherence on CHI/HA films}

Staining cells with crystal violet was performed to quantify adherent cells after 2 weeks of culture. For $(\mathrm{CHI} / \mathrm{HA})_{10}$ 

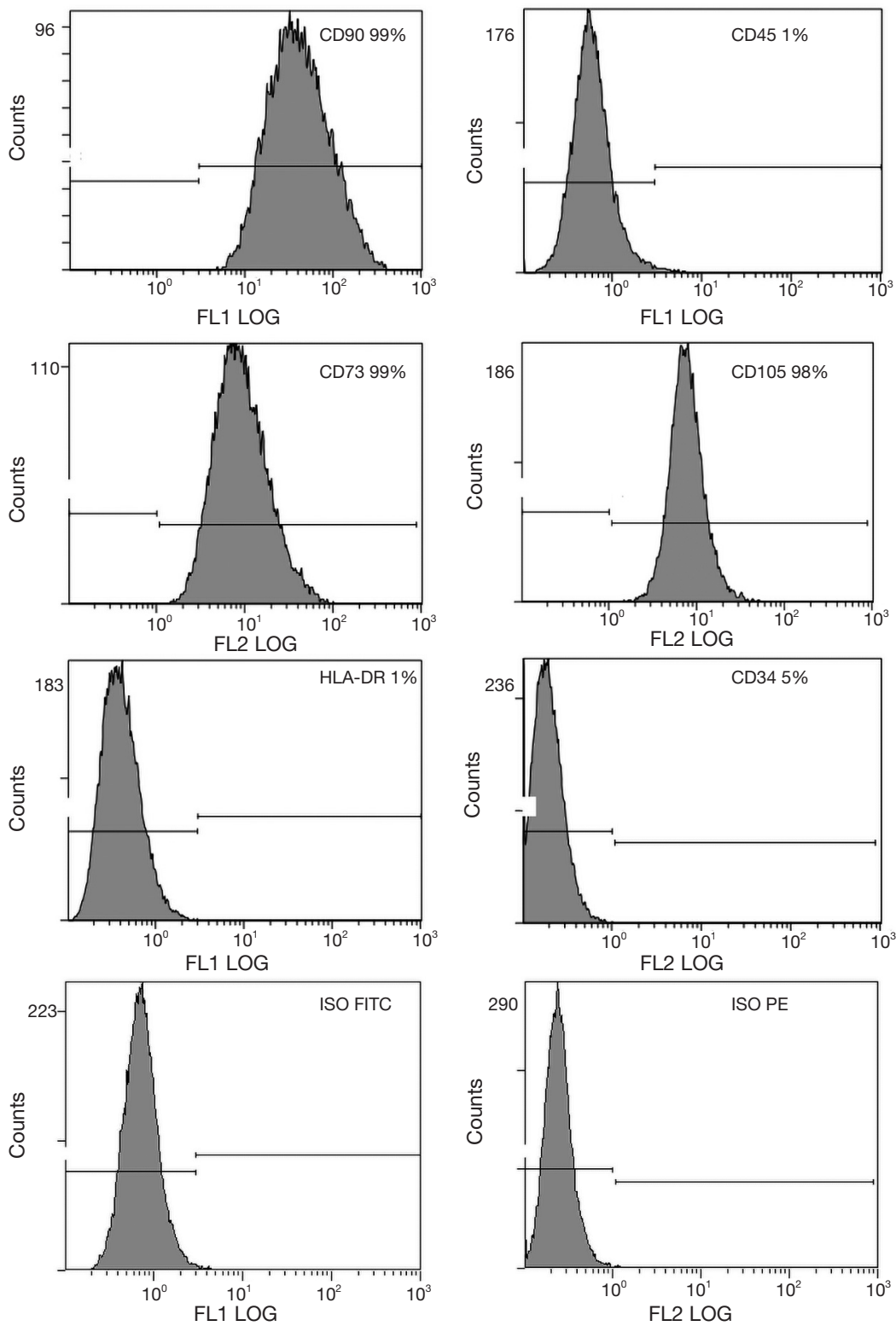

Figure 1 Characterization of mesenchymal stem cells (MSCs). MSCs isolated from Wharton's jelly of Human Umbilical Cords were characterized by flow cytometry. The gray histograms represent test samples. Inset value denotes the percentage of the positive population. MSCs were positive for cluster of differentiation 90 (CD90), CD105, and CD73; and were negative for CD34, HLA-DR and CD45.

we got relatively close and better absorbance values than for collagen I (Figure 2), indicating that the cells were well adhered on $(\mathrm{CHI} / \mathrm{HA})_{10}$. The numeration of nuclei showed an important cell adhesion on (CHI/HA $)_{10}$ compared to the positive control: collagen I (Figure 3). These results confirmed those obtained with dye crystal violet.

\section{Evaluation of CHI/HA films integrity}

The immunostaining of WJ-MSCs seeded on collagen I and $(\mathrm{CHI} / \mathrm{HA})_{10}$ showed spread actin microfilament formation and a fibroblast-like morphology. After two weeks, the cells reach their confluence on these polyelectrolyte multilayer films compared to the positive control cells seeded on 


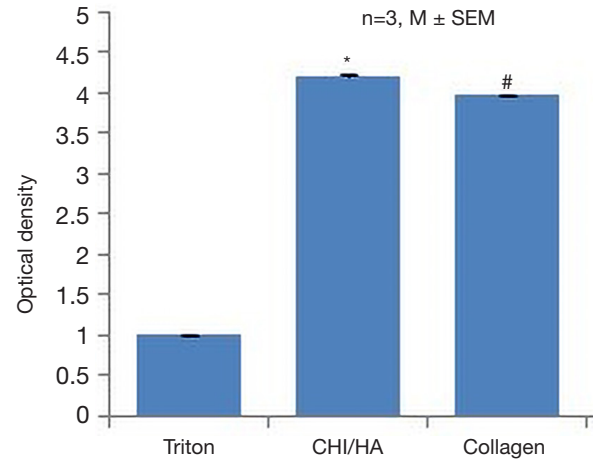

Figure 2 Cell adherence, Wharton's jelly (WJ) derived mesenchymal stem cells (MSCs) adherence was determined by Crystal violet after 2 weeks of culture on Chitosan/hyaluronic acid (CHI/HA) and collagen substrates. The extent of cell adhesion was quantified by measuring the optical density of the eluted crystal violet at $570 \mathrm{~nm}$. Triton treated cells then stained with Crystal violet were used as negative control. Results are expressed $\pm \mathrm{SEM}$, $\mathrm{n}=3$. *, Triton versus CHI/HA; ", Triton versus Collagen, $\mathrm{P}<0.01$.

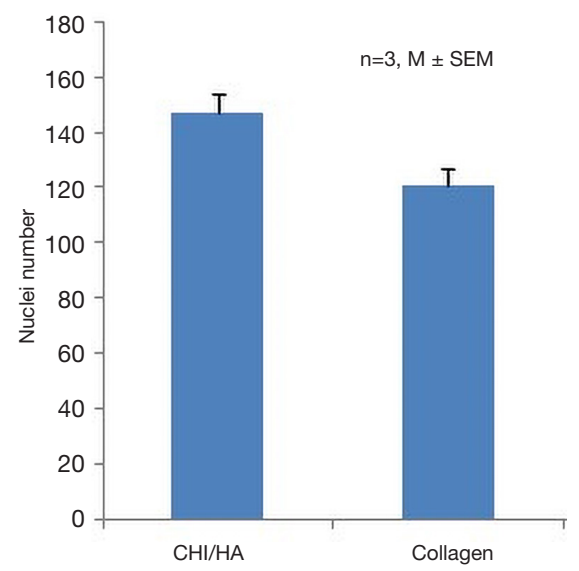

Figure 3 Cell adherence, Wharton's jelly (WJ) derived mesenchymal stem cells (MSCs) adherence was determined by counting the nuclei number after 2 weeks of culture on CHI/HA and collagen substrates. Results are expressed \pm SEM, $n=3$.

collagen I matrix (Figure 4). Photos obtained with SEM showed that cells grown on (CHI/HA) $)_{10}$ exhibit fibroblastlike morphology characteristic of MSCs and identical to that of the control side (Figure 5). These observations confirmed cytoskeleton staining results. So on CHI/HA films, WJMSCs could adhere and proliferate in a monolayer aspect conserving the fibroblast-like morphology.
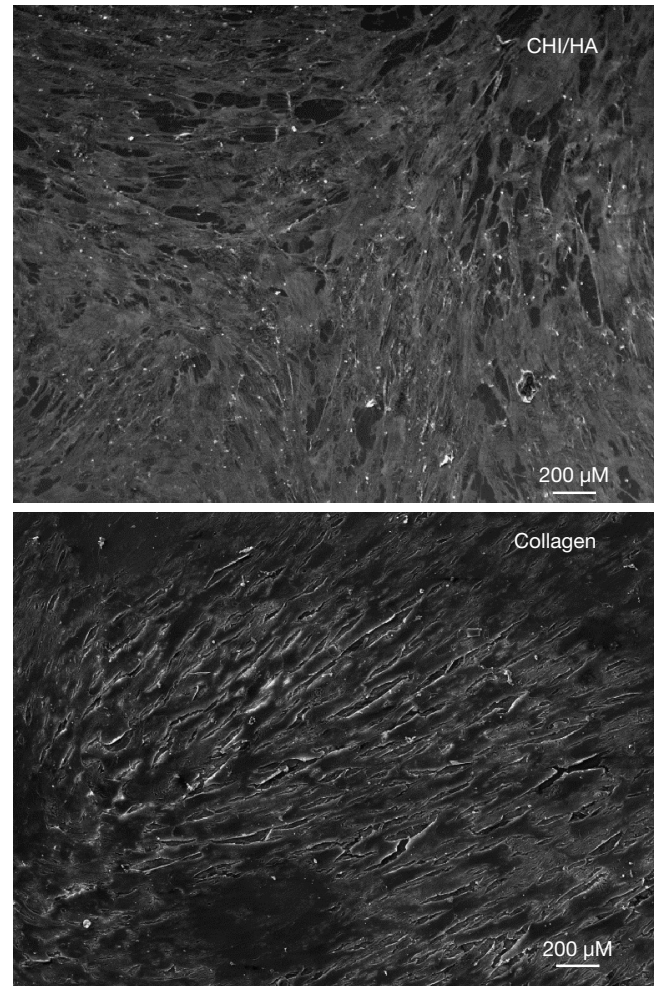

Figure 4 SEM, Photos of Wharton's jelly derived mesenchymal stem cells (WJ-MSCs) two weeks after seeding on Chitosan/ hyaluronic acid (CHI/HA) and collagen by Scanning Electron Microscopy (JEOL Phillips Microscope, Objective ×150). SEM, Scanning Electron Microscopy.

\section{Discussion}

Cell scaffolds made up of natural polysaccharides have attracted significant attention because of their various biocompatibilities and specific biological functions based on their chemical structures $(23,24)$. Chitosan obtained by the deacetylation of chitin, has been widely used solely or combined with other polysaccharides, because it is a basic polymer rarely found in natural polysaccharides and is soluble in weak acid, which allows for easier handling (25). Cell scaffolds of CHI composited with glycosaminoglycans (GAGs), one of the major components of the cartilage extracellular matrix (ECM), have been developed for cartilage tissue engineering $(26,27)$. Cultures of chondrocytes on composite scaffolds made of chondroitin sulfate $\mathrm{C}(\mathrm{CS})$, dermatan sulfate, and $\mathrm{CHI}$, mimicking the ECM of natural cartilage, were found to maintain chondrocyte differentiation while suppressing de- 

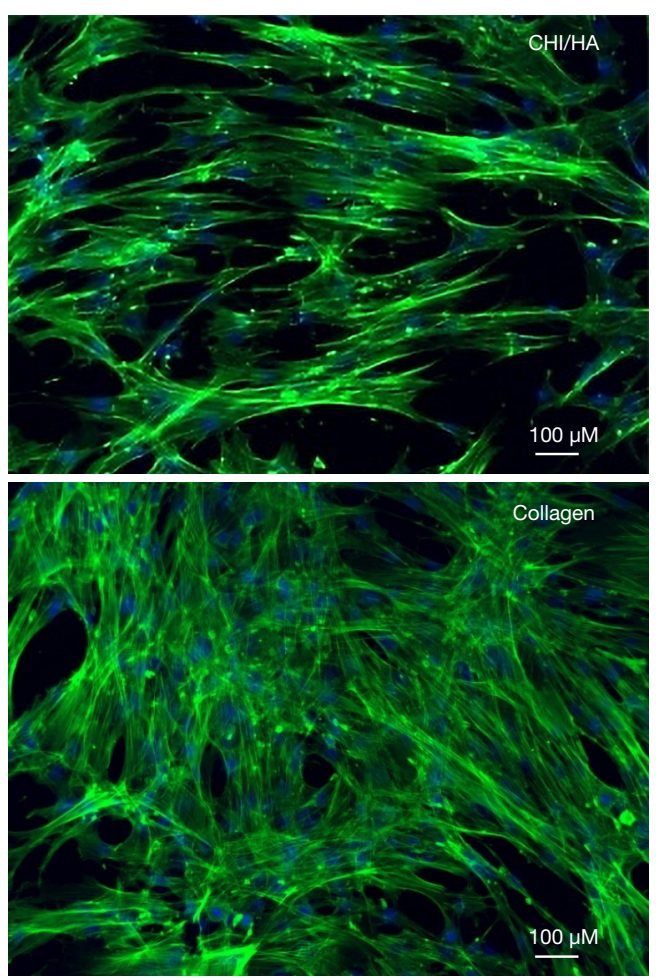

Figure 5 Cytoskeleton staining, immunostaining of $\alpha$-actin filaments (green), and nuclei staining with DAPI (blue) of WJMSCs, after two weeks of culture in $\alpha-M E M$ on CHI/HA and collagen (Zeiss Microscope, Objective $\times 20$ ).

differentiation and hypertrophy (23). Composite scaffolds made up of GAGs and $\mathrm{CHI}$ have also been used for bone tissue engineering because GAGs modulate the activities of several cytokines and growth factors that are important for bone regeneration (28).

Since hyaluronic acid, a non-sulfonated GAG in the ECM, regulates cancer stemness and tumor progression (29), HYA-based hydrogels have been used in the reconstitution of tumor microenvironments in vitro (30). Furthermore, a scaffold made of alginic acid (ALG) and CHI enabled feeder-free self-renewal of human embryonic stem cells (26).

Therefore, cell scaffolds made of polysaccharides have potential not only as materials for implantable scaffolds, but also tools for cancer research and the ex vivo expansion of stem cells for regenerative medicine.

Different studies showed that MSCs behavior is highly dependent on physicochemical properties of the culture substrate (matrix, scaffold... c'est mieux?). Literature has shown that collagen coating allowed an effective MSCs adhesion but it's essential for platelet aggregation and activation (31). Moreover, fibronectin coated surfaces had enhancing effect on cell adhesion, however it suffers from being hydrolyzed and so there is a need for another substrate for clinical use (32).

In this report, we have used $(\mathrm{CHI} / \mathrm{HA})_{10}$ films as substrate for WJ-MSCs adhesion and proliferation. Before seeding WJ-MSCs on this scaffold, we have examined their immune phenotype. No single marker was identified so far predictably defining MSC. Traditionally culture expanded MSCs are described to be negative for hematopoietic markers including CD45 and CD34. On the contrary MSCs express CD44, CD73, CD90 and CD105. In addition, MSCs are described by the absence of HLA class II $(33,34)$. Indeed our cells satisfied this panel.

Cell adherence was evaluated using crystal violet and nuclei staining. Results showed a great biocompatibility of $(\mathrm{CHI} / \mathrm{HA})_{10}$ for WJ-MSCs that adhere and proliferate for 2 weeks of culture compared to collagen I.

Actin cytoskeleton was labeled to evaluate cell morphology of WJ-MSCs cultured on (CHI/HA $)_{10}$ and collagen I coatings after 2 weeks. The cytoskeleton showed that WJ-MSCs cultured on (CHI/HA $)_{10}$ and collagen I coatings presented a typical fibroblast cell shape with well-aligned actin fibers that span over the entire cell body. Reorganization of actin stress fibers actively participates in cell signal transduction and transmits signals from extracellular matrix to nucleus to influence gene expression $(18,31,35)$. Photos obtained with SEM showed that cells grown on (CHI/HA) ${ }_{10}$ exhibit fibroblast-like morphology characteristic of MSCs and identical to that of the control side. These observations confirmed cytoskeleton staining results.

These multilayer CHI/HA films have been described as poorly adhesive for chondrosarcoma and osteoblastic cell line (14) but showed a good human fibroblasts and bone marrow mesenchymal stem cells (BM-MSCs) adhesion and proliferation $(10,15)$. In this study we have shown that formation of PEMs from CHI and HA resulted in surfaces that promoted WJ-MSCs adhesion and proliferation.

\section{Conclusions}

These promising results showed that WJ-MSCs could adhere and proliferate in a monolayer aspect conserving the fibroblast-like morphology on CHI/HA multilayer films.

\section{Acknowledgements}

The authors would like to thank Al Hanan Hospital for 
providing the umbilical cords used in our researches and Azm \& Saadeh society for funding this work.

\section{Footnote}

Conflicts of Interest: The authors have no conflicts of interest to declare.

Ethical Statement: The research work was approved by the ethical committee of the Lebanese University, Centre Azm for research in Applied Biotechnology and the ethical committee of Al Hanan Hospital-Tripoli, Lebanon.

\section{References}

1. Gribova V, Auzely-Velty R, Picart C. Polyelectrolyte multilayer assemblies on materials surfaces: from cell adhesion to tissue engineering. Chem Mater 2012;24:854-69.

2. Moby V, Boura C, Kerdjoudj H, et al. Poly (styrenesulfonate)/poly (allylamine) multilayers: a route to favor endothelial cell growth on expanded poly (tetrafluoroethylene) vascular grafts. Biomacromolecules 2007;8:2156-60.

3. Thompson MT, Berg MC, Tobias IS, et al. Tuning compliance of nanoscale polyelectrolyte multilayers to modulate cell adhesion. Biomaterials 2005;26:6836-45.

4. Berthelemy N, Kerdjoudj H, Gaucher C, et al. Polyelectrolyte Films Boost Progenitor Cell Differentiation into Endothelium-like Monolayers. Adv Mater 2008;20:2674-8.

5. Salmon N, Paternotte E, Decot V, et al. Polyelectrolyte multilayer films promote human cord blood stem cells differentiation into mature endothelial cells exhibiting a stable phenotype. Biomed Mater Eng 2009;19:349-54.

6. Kerdjoudj H, Boulmedais F, Berthelemy N, et al. Cellularized alginate sheets for blood vessel reconstruction. Soft Matter 2011;7:3621-6.

7. Newcomer RG, Moussallem MD, Keller T, et al. Human coronary artery smooth muscle cell responses to bioactive polyelectrolyte multilayer interfaces. Biotechnol Res Int 2011;2011:854068.

8. Boura C, Muller S, Vautier D, et al. Endothelial cellinteractions with polyelectrolyte multilayer films. Biomaterials 2005;26:4568-75.

9. Picart C. Polyelectrolyte multilayer films: from physicochemical properties to the control of cellular processes. Curr Med Chem 2008;15:685-97.
10. Cado G, Aslam R, Séon L, et al. Self-Defensive Biomaterial Coating Against Bacteria and Yeasts: Polysaccharide Multilayer Film with Embedded Antimicrobial Peptide. Adv Funct Mater 2013;23:4801-9.

11. Cado G, Kerdjoudj H, Chassepot A, et al. Polysaccharide films built by simultaneous or alternate spray: a rapid way to engineer biomaterial surfaces. Langmuir 2012;28:8470-8.

12. Petzold G, Petzold-Welcke K, Qi H, et al. The removal of stickies with modified starch and chitosan-Highly cationic and hydrophobic types compared with unmodified ones. Carbohydr Polym 2012;90:1712-8.

13. Hillberg AL, Holmes CA, Tabrizian M. Effect of genipin cross-linking on the cellular adhesion properties of layerby-layer assembled polyelectrolyte films. Biomaterials 2009;30:4463-70.

14. Richert L, Lavalle P, Payan E, et al. Layer by layer buildup of polysaccharide films: physical chemistry and cellular adhesion aspects. Langmuir 2004;20:448-58.

15. Liu ZM, Gu Q, Xu ZK, et al. Synergistic effect of polyelectrolyte multilayers and osteogenic growth medium on differentiation of human mesenchymal stem cells. Macromol Biosci 2010;10:1043-54.

16. Medicetty S, Bledsoe AR, Fahrenholtz CB, et al. Transplantation of pig stem cells into rat brain: Proliferation during the first 8 weeks. Exp Neurol 2004;190:32-41.

17. Patel DM, Shah J, Srivastava AS. Therapeutic potential of mesenchymal stem cells in regenerative medicine. Stem Cells Int 2013;2013:496218.

18. Pittenger MF, Martin BJ. Mesenchymal stem cells and their potential as cardiac therapeutics. Circ Res 2004;95:9-20.

19. Wang S, Qu X, Zhao RC. Clinical applications of mesenchymal stem cells. J Hematol Oncol 2012;5:19.

20. La Rocca G, Lo Iacono M, Corsello T, et al. Human Wharton's jelly mesenchymal stem cells maintain the expression of key immunomodulatory molecules when subjected to osteogenic, adipogenic and chondrogenic differentiation in vitro: new perspectives for cellular therapy. Curr Stem Cell Res Ther 2013;8:100-13.

21. Fu YS, Cheng YC, Lin MY, et al. Conversion of human umbilical cord mesenchymal stem cells in Wharton's jelly to dopaminergic neurons in vitro: potential therapeutic application for Parkinsonism. Stem Cells 2006;24:115-24.

22. Rammal H, Harmouch C, Maerten C, et al. Upregulation of endothelial gene markers in Wharton's jelly mesenchymal stem cells cultured on polyelectrolyte multilayers. J Biomed Mater Res A 2017;105:292-300. 
23. Asti A, Gioglio L. Natural and synthetic biodegradable polymers: different scaffolds for cell expansion and tissue formation. Int J Artif Organs 2014;37:187-205.

24. Iijima K, Hashizume M. Application of polysaccharides as structural materials. Trends Glycosci Glycotechnol 2015;27:67-79.

25. Rodríguez-Vázquez M, Vega-Ruiz B, Ramos-Zúñiga R, et al. Chitosan and its potential use as a scaffold for tissue engineering in regenerative medicine. Biomed Res Int 2015;2015:821279.

26. Li Z, Leung M, Hopper R, et al. Feeder-free self-renewal of human embryonic stem cells in $3 \mathrm{D}$ porous natural polymer scaffolds. Biomaterials 2010;31:404-12.

27. Suh J-KF, Matthew HW. Application of chitosan-based polysaccharide biomaterials in cartilage tissue engineering: a review. Biomaterials 2000;21:2589-98.

28. Levengood SL, Zhang M. Chitosan-based scaffolds for bone tissue engineering. J Mater Chem B 2014;2:3161-84.

29. Chanmee T, Ontong P, Itano N. Hyaluronan: A modulator of the tumor microenvironment. Cancer Lett 2016;375:20-30.

\section{doi: $10.21037 /$ sci.2018.12.02}

Cite this article as: Dennaoui $\mathrm{H}$, Chouery E, Rammal H, Abdel-Razzak Z, Harmouch C. Chitosan/hyaluronic acid multilayer films are biocompatible substrate for Wharton's jelly derived stem cells. Stem Cell Investig 2018;5:47.
30. Xu X, Gurski LA, Zhang C, et al. Recreating the tumor microenvironment in a bilayer, hyaluronic acid hydrogel construct for the growth of prostate cancer spheroids. Biomaterials 2012;33:9049-60.

31. Weinberg CB, Bell E. A blood vessel model constructed from collagen and cultured vascular cells. Science. 1986;231:397-400.

32. Tiwari A, Salacinski HJ, Punshon G, et al. Development of a hybrid cardiovascular graft using a tissue engineering approach. FASEB J 2002;16:791-6.

33. Carvalho MM, Teixeira FG, Reis RL, et al. Mesenchymal stem cells in the umbilical cord: phenotypic characterization, secretome and applications in central nervous system regenerative medicine. Curr Stem Cell Res Ther 2011;6:221-8.

34. Margossian T, Reppel L, Makdissy N, et al. Mesenchymal stem cells derived from Wharton's jelly: comparative phenotype analysis between tissue and in vitro expansion. Biomed Mater Eng 2012;22:243-54.

35. Murphy WL, McDevitt TC, Engler AJ. Materials as stem cell regulators. Nat Mater 2014;13:547. 